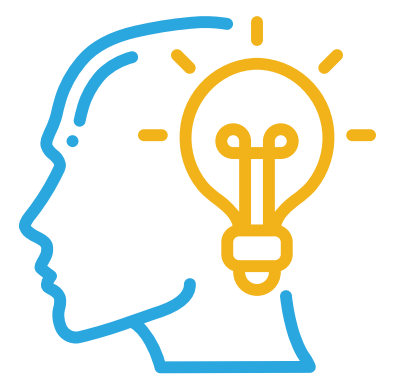

\title{
MOSAICOS DO PENSAR E DO FAZER: CONSTRUINDO UMA PROPOSTA DE ENFRENTAMENTO À SÍFILIS
}

\author{
MOSAICS OF THINKING AND DOING: BUILDING A PROPOSAL TO \\ CONFRONTING SYPHILIS
}

\begin{abstract}
Sofia Campos dos Santos
Terapeuta Ocupacional, Pedagoga, Especialista em Saúde do Trabalhador, Técnica da Secretaria Municipal de Saúde de Salvador, Referência para Sífilis da Vigilância Epidemiológica/Setor de Acompanhamento das

IST. Apoiadora de Pesquisa e Intervenção do Projeto Sífilis Não. E-mail: sofia.santos@lais.huol.ufrn.br.

\section{Vania Priamo}

Fisioterapeuta, Mestra em Saúde Coletiva, com Residência em Saúde da Família, Especialista em Educação

Permanente em Saúde e Ativadores de Mudança da Formação Profissional em Saúde. Apoiadora de Núcleo da Residência Multiprofissional em Saúde da Família. Apoiadora de Pesquisa e Intervenção do Projeto Sífilis Não. E-mail: vania.priamo@lais.huol.ufrn.br.

Jamile Soares dos Santos

Assistente Social, Pedagoga, Especialista em Atenção Integral ao Consumo e Consumidores de álcool e outras drogas, Especialista em Serviço Social, Saúde e Contemporaneidade. Apoiadora de Pesquisa e Intervenção do Projeto Sífilis Não. E-mail: jamile.santos@lais.huol.ufrn.br.

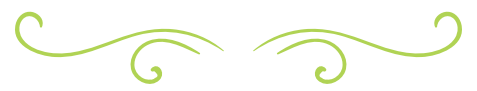

\section{RESUMO}

O objetivo deste artigo é descrever o processo de elaboração de uma proposta do Plano Municipal de Enfrentamento da Sífilis. As apoiadoras puderam vivenciar as etapas de construção do documento, por meio do acompanhamento de diferentes atividades com diversos setores da Secretaria Municipal da Saúde (SMS). O estudo observou aspectos relevantes de um trabalho coletivo, intersetorial e multiprofissional, bem como foi uma oportunidade de avaliar o papel do apoiador e a importância das contribuições do Projeto "Sífilis Não" para a implantação/ implementação de ações para combate ao agravo. A metodologia utilizada para construção do "Plano da Sífilis" tem por base o planejamento estratégico em saúde, adotado pela Secretaria Municipal de Saúde desde 2014. Esse processo resultou, além da proposta de um plano, na implantação da Câmara Técnica Municipal de Prevenção da Transmissão Vertical da Sífilis, HIV, HTLV e Hepatites Virais.

Palavras-chave: Planejamento; Sífilis; Projeto "Sífilis Não"; Apoio. 


\section{ABSTRACT}

The objective of this paper is to describe the experience for the elaboration of a Municipal Syphilis Confronting Plan. The supporters were able to experience all the stages of this document construction by monitoring different activities with several Municipal Health Secretariat (SMS) sectors. It was possible to observe relevant aspects of collective, intersectoral and multiprofessional work, as well as an opportunity to evaluate the supporter's roles and the No Syphilis Project importance contributions for the implementation of actions to combat the problem. The methodology used to architect the "Syphilis Plan" was based on Strategic Health Planning (PES), adopted by the Municipal Health Secretariat since 2014. This process resulted, in addition to the proposal for a plan, in the Municipal Technical Chamber for the Prevention of Vertical Transmission of Syphilis, HIV, HTLV and Viral Hepatitis implementation (CPTV).

Key-words: Planning; Syphilis; Project Syphilis No; Support.

\section{INTRODUÇÃO}

As Infecções Sexualmente Transmissíveis (IST) constituem um desafio para os serviços de saúde do mundo. A sífilis é uma IST causada pelo Treponema pallidum. Pode ser adquirida através de contato sexual, transfusão de sangue ou por transmissão vertical, que é o modo de transmissão de maior impacto para a saúde pública, devido à frequência dos desfechos graves para a gestante e para a criança - parto prematuro, óbito fetal e neonatal e infecção congênita do recém-nascido.

A redução da sífilis e a eliminação da sífilis congênita é um problema de saúde pública que exige esforços dos gestores, profissionais de diferentes áreas, principalmente da saúde, e da sociedade civil. O diagnóstico e o tratamento estão disponíveis no Sistema Único de Saúde (SUS), entretanto, ainda não foi possível reduzir a incidência de sífilis nem eliminar a transmissão da gestante infectada para o feto (sífilis congênita). Desde 1992, o Brasil é signatário de compromissos internacionais para eliminação da sífilis congênita. Em 2016, a sífilis, em todas as suas formas, foi declarada como um grave problema de saúde pública no Brasil, sobretudo entre homens e nos segmentos mais jovens da população brasileira (BRASIL, 2018).

Visando ao controle da epidemia de sífilis, o Departamento de Vigilância, Prevenção e Controle das Infecções Sexualmente Transmissíveis (IST), do HIV/Aids e das Hepatites Virais, da Secretaria de Vigilância em Saúde, do Ministério da Saúde (DIAHVI MS/SVS), elaborou uma Agenda de Ações Estratégicas para Redução da sífilis no Brasil, "que resultou na iniciativa de uma emenda parlamentar de 200 milhões de reais, destinada a implementar um projeto de resposta rápida à sífilis em 100 municípios prioritários, que respondem por aproximadamente 65\% dos casos de sífilis do país" (BRASIL, 2018).

O projeto, denominado "Sífilis Não", é uma iniciativa do Ministério da Saúde em parceria com a Universidade Federal do Rio Grande do Norte (UFRN) - Coordenação do Laboratório de Inovação Tecnológica em Saúde (LAIS) e apoio técnico do Núcleo de Estudos em Saúde Coletiva (NESC) - com a participação de 52 apoiadores locais, a partir de abril de 2018, visando ao fortalecimento das redes de atenção à saúde e vigilância em saúde para controle da sífilis, além da produção de pesquisas científicas e ações de comunicação e educação em saúde.

Durante a elaboração da cartografia do território, ao realizar o levantamento dos documentos de gestão, as apoiadoras identificaram a existência de um plano municipal para a sífilis, elaborado em 2011. No entanto, além das fragilidades observadas na execução das propostas, os gestores afirmavam que era necessário um plano atualizado e em consonância com o modelo de planejamento adotado pela SMS. Ao longo da história do Sistema Único de Saúde (SUS), 
já é reconhecida a importância do planejamento para a organização e o fortalecimento das ações de saúde para aumentar a resolutividade dos serviços (BRANDÃO, 2019).

De acordo com informações registradas no Plano Municipal de Saúde 2018-2021 (SALVADOR, 2017), o município de Salvador situa-se no Recôncavo Baiano, com uma população de aproximadamente 2.938.092 habitantes, distribuídos em 692,8 km2 e densidade demográfica de 3.859,4 hab/km2 (IBGE, 2010). É a quarta capital do país em termos populacionais e compõe a Região Metropolitana de Salvador (RMS) com mais 12 municípios. A estrutura político-administrativa de Salvador compreende 10 regiões administrativas (prefeituras bairros) e 12 Distritos Sanitários (DS) e se situa no Núcleo Regional de Saúde Leste.

Do ponto de vista dos condicionantes e determinantes sociais, a distribuição da população é marcada por grandes contrastes, com uma orla marítima organizada e melhores condições de vida, ao passo que as regiões centrais e periféricas são densamente povoadas e vivendo em condições precárias.

Analisando os dados epidemiológicos relacionados à sífilis, verificou-se que, em Salvador, a sífilis adquirida teve sua taxa de detecção aumentada de 0 caso, em 2010, para 100,6 casos por 100.000 habitantes, em 2019. No mesmo período, a taxa de detecção de sífilis em gestantes variou de 4,2 para 36,6 por 1.000 nascidos vivos; e a taxa de incidência de sífilis congênita foi de 2,9 para 16,8 por 1.000 nascidos vivos. Tanto a sífilis adquirida como a sífilis em gestantes atingem, principalmente, adultos jovens e adolescentes, pardos e pretos, cuja escolaridade está entre o Ensino fundamental incompleto e o Ensino Médio (SALVADOR, 2020).

Diante do impacto da sífilis para a saúde sexual e reprodutiva das pessoas e para a saúde de milhares de crianças e considerando a relevância do planejamento e a organização das ações nos serviços de saúde, é mister o relato da experiência singular das apoiadoras de pesquisa e intervenção do Projeto "Sífilis Não", no processo de elaboração da proposta do Plano Municipal de Enfrentamento da Sífilis em Salvador, Bahia.

Nesse sentido, o presente trabalho tem o objetivo de descrever a experiência de apoio para a elaboração de uma proposta do Plano Municipal de Enfrentamento da Sífilis. As apoiadoras puderam vivenciar as etapas de construção do documento, pelo acompanhamento de diferentes atividades com diversos setores da Secretaria Municipal da Saúde (SMS).

\section{METODOLOGIA}

Desde o início das atividades do apoio, optou-se por um apoio integrado independente do território de atuação das apoiadoras. $\mathrm{Ou}$ seja, as intervenções seriam realizadas pelas três apoiadoras nos dois municípios, do Estado da Bahia.

A perspectiva adotada para o apoio visou ampliar a capacidade dos sujeitos individuais e coletivos para gestão, análise e intervenção sobre determinada realidade. O papel do apoiador baseou-se na articulação, mediação, negociação e utilização de instrumentos de gestão como o planejamento, a avaliação e o monitoramento das atividades cotidianas. Segundo Campos (2010), o apoio é um método que possibilita aos sujeitos refletir sobre o fazer e buscar novos saberes e (re)criar o trabalho em saúde. Nesse sentido, o planejamento das ações torna-se uma construção coletiva, em espaços democráticos de fala e escuta.

Para a construção do Plano Municipal de Enfrentamento da Sífilis, a metodologia utilizada baseou-se no planejamento estratégico em saúde (PES), adotada pela SMS desde 2014. O planejamento, assim, deve ser entendido como um modo de explicitar a Política de Saúde, definindo o que vai ser feito, quando, onde, como e com quem, numa perspectiva de mobilizar vontades, de uma ação coletiva e compartilhada, 
podendo promover o aumento da mobilização política e da consciência sanitária dos atores envolvidos (CERQUEIRA et al., 2020).

No caso das instituições de saúde, em que a quantidade e a complexidade das tarefas a serem realizadas, bem como o volume de recursos e pessoas envolvidas na sua realização não podem correr o risco do improviso, a necessidade de planejamento torna-se premente. Acresce-se a isso o fato de lidarem com situações que envolvem a vida de milhões de pessoas e que podem resultar em doenças, incapacidades e mortes (PAIM, 2006, p. 767).

A proposta do Plano Municipal de Enfrentamento da Sífilis teve como objetivo a implementação de ações estratégicas para prevenção; detecção precoce; redução da infecção da sífilis em homens e mulheres; eliminação da transmissão vertical no pré-natal e no parto e garantia do acompanhamento da puérpera e do recém-nascido.

Inicialmente foi realizada a análise dos instrumentos de gestão - Plano Municipal de Saúde (PMS), Programação Anual de Saúde (PAS), Programação Operacional Anual dos Distritos Sanitários (POA) e do Plano Municipal para Enfrentamento da Sífilis, elaborado em 2011 - visando identificar a inclusão de ações de combate à sífilis. $\mathrm{Na}$ oportunidade, foi realizado o levantamento de problemas apontados a partir das oficinas para construção do Plano Municipal de Saúde de Salvador (2018-2021). Os documentos foram disponibilizados pela Chefia do Setor de Monitoramento e Acompanhamento das IST, HIV/Aids e Hepatites Virais, ligado à Diretoria de Vigilância em Saúde (DVIS).

Em seguida, foram realizadas visitas aos 12 Distritos Sanitários (DS) e aos 03 Serviços Especializados (SAE), com o intuito de identificar fatores que pudessem contribuir para a proposta do plano, incluindo levantamento de potencialidades e fragilidades que cada DS apontava para o desenvolvimento de ações de enfrentamento à sífilis. Essas visitas foram realizadas entre os meses de setembro e outubro de 2018, em conjunto com as técnicas de referência para sífilis, HTLV e Hepatites Virais e 01 técnica da Diretoria de Assistência à Saúde (DAS). A técnica de referência para HIV/Aids só pode participar de uma visita.

Foi realizado o Encontro Municipal de Enfrentamento da Sífilis - 23 de outubro de 2018, que reuniu representantes da gestão estadual e municipal e trabalhadores dos distritos sanitários. Durante o evento, foi apresentada a situação epidemiológica da sífilis mundialmente, no Brasil, na Bahia e em Salvador; foi apresentado o Projeto de Resposta Rápida à Sífilis nas Redes de Atenção; e, também, foram expostos alguns trabalhos de conclusão do EpiSUS Fundamental sobre experiências municipais para enfrentamento à sífilis.

Ao final do encontro, foi apresentada a proposta do planejamento de ações a ser realizadas por meio de oficinas distritais, contando com a participação dos trabalhadores da saúde, Nível Central e DS, e Agentes Comunitários em Saúde (ACS). Ficou decidida a realização de 8 oficinas distritais, em alguns casos, com dois distritos, que apresentavam proximidade geográfica e epidemiológica. As oficinas, sob coordenação das apoiadoras e uma técnica do Setor de IST, ocorreram entre os meses de novembro e dezembro de 2018, com a produção de uma matriz de planejamento que foi avaliada quadrimestralmente a partir de janeiro de 2019. Entre o $2^{\circ}$ e $3^{\circ}$ quadrimestre de 2019, houve mais uma rodada de visita aos 12 Distritos Sanitários, quando foi possível avaliar e revisar as ações propostas no ano anterior.

Importante ressaltar que, a partir desse movimento de aproximação com os DS, o Grupo de Trabalho Municipal da Sífilis, existente desde 2015, cuja composição estava restrita aos representantes do Nível Central da SMS (Assistência e Vigilância) e apenas 01 representante de DS, ampliou seus componentes para garantir a representatividade dos atores que estavam mobilizados na pauta da sífilis: Atenção Primária; Saúde da Criança, da 
Mulher, do Homem; Rede Cegonha; Campo Temático LGBTQ+; Atenção Especializada. A ampliação do GT Sífilis possibilitou a avaliação das ações de prevenção, diagnóstico e tratamento da sífilis e reflexão sobre a necessidade de construção da Linha do Cuidado para pessoas com sífilis, crianças expostas e com sífilis congênita.

Para a análise da situação epidemiológica da sífilis no município, no período de 2010 a 2019, foram utilizados dados disponíveis no SINAN; TabWin, além dos Boletins Epidemiológicos da Sífilis do Ministério da Saúde, da Secretaria Estadual de Saúde e da SMS, dos anos 2018 e 2019. Os resultados encontrados no município trouxeram um cenário com a taxa de detecção de sífilis adquirida e em gestantes e a taxa de incidência de sífilis congênita maiores que a do Brasil, Nordeste e Bahia nos períodos analisados. A sífilis adquirida teve sua taxa de detecção aumentada de 0 caso, em 2010, para 100,6 casos por 100.000 habitantes, em 2019. No mesmo período, a taxa de detecção de sífilis em gestantes variou de 4,2 para 36,6 por 1.000 nascidos vivos e a taxa de incidência de sífilis congênita foi de 2,9 para 16,8 por 1.000 nascidos vivos (Gráfico 1).

Gráfico 1 - Taxa de detecção de sífilis adquirida, taxa de detecção de sífilis em gestante e taxa de incidência de sífilis congênita, Salvador, 2010-2019.

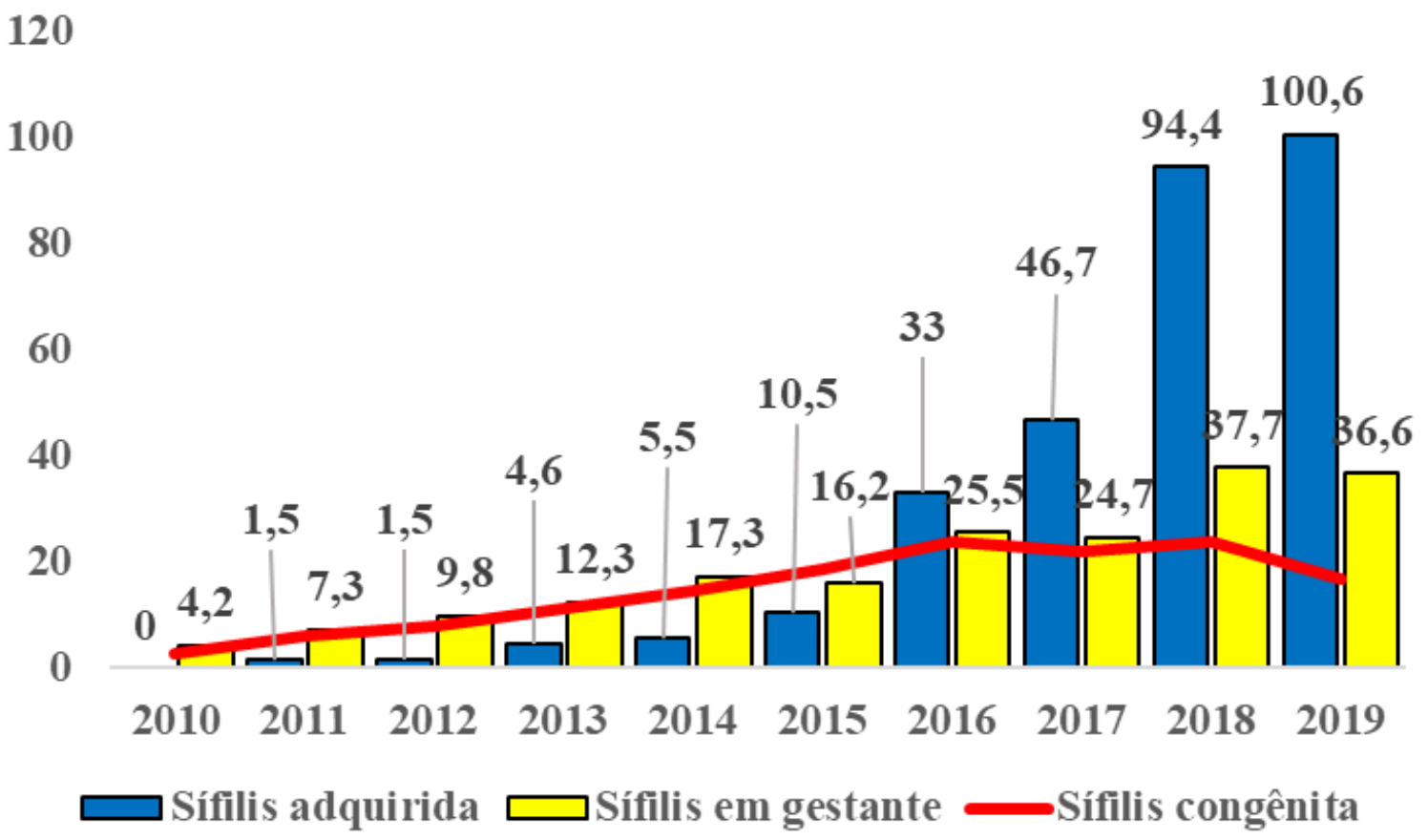

Fonte: SMS/DVIS/Viep/IST-Tabwin/SinanNet. Acesso em 30 jun/20. Sujeito a alteração

Em Salvador, entre 2010 e 2019, os Distritos Sanitários (DS) com maiores taxas de detecção de sífilis adquirida foram Centro Histórico, Pau da Lima e Barra/Rio Vermelho. Em 2019, as taxas de detecção dos referidos DS foram respectivamente, 269,9/100000 habitantes; 172,7/100000 habitantes e 154,4/100000 habitantes. Entre 2018 e 2019, apenas o DS Cajazeiras apresentou redução da taxa de detecção de sífilis adquirida, passando de 127,2/100000 habitantes, em 2018, para 113,8/100000 habitantes, em 2019. (Tabela 1). 
Tabela 1 - Taxa de detecção de casos confirmados de sífilis adquirida por distrito sanitário, Salvador, 2010-2019.

\begin{tabular}{|c|c|c|c|c|c|c|c|c|c|}
\hline $\begin{array}{l}\text { Distrito } \\
\text { Sanitário }\end{array}$ & 2011 & 2012 & 2013 & 2014 & 2015 & 2016 & 2017 & 2018 & 2019 \\
\hline $\begin{array}{l}\text { Centro } \\
\text { Histórico }\end{array}$ & 1,4 & 2,7 & 7,7 & 9 & 20,4 & 115,3 & 141,1 & 153,7 & 269,9 \\
\hline Itapagipe & 1,8 & 0 & 3,4 & 7,9 & 6,8 & 30,8 & 37,9 & 52,5 & 148,7 \\
\hline $\begin{array}{c}\text { São } \\
\text { Caetano/ } \\
\text { Valeria }\end{array}$ & 1,2 & 1,9 & 1,4 & 2,1 & 12,5 & 24,4 & 46,8 & 82,6 & 129,3 \\
\hline Liberdade & 2,2 & 1,7 & 0,5 & 3,1 & 3,6 & 19,3 & 80,8 & 81,9 & 83,7 \\
\hline Brotas & 1 & 0,5 & 0,5 & 1,4 & 5 & 46,1 & 57,9 & 72,3 & 109,6 \\
\hline $\begin{array}{c}\text { Barra/R } \\
\text { Vermelho }\end{array}$ & 0,9 & 0 & 4,9 & 4,9 & 5,9 & 24,5 & 35,8 & 88,1 & 154,4 \\
\hline $\begin{array}{l}\text { Boca do } \\
\text { Rio }\end{array}$ & 2,4 & 3,1 & 2,9 & 3,6 & 13,8 & 35,3 & 51,6 & 97,1 & 110,7 \\
\hline Itapuã & 1,2 & 0,4 & 1,9 & 3,3 & 3,7 & 16 & 34,4 & 82,7 & 115,5 \\
\hline $\begin{array}{c}\text { Cabula/ } \\
\text { Beiru }\end{array}$ & 1,8 & 1,3 & 3,1 & 11,2 & 28 & 51,2 & 46 & 116,5 & 150,3 \\
\hline $\begin{array}{l}\text { Pau da } \\
\text { Lima }\end{array}$ & 2,3 & 2,3 & 0,4 & 10,1 & 6,3 & 44,6 & 43,1 & 139,2 & 172,7 \\
\hline $\begin{array}{l}\text { Subúrbio } \\
\text { Ferroviário }\end{array}$ & 0,3 & 0 & 4 & 3,7 & 7,7 & 20,4 & 28,1 & 64,2 & 136,1 \\
\hline Cajazeiras & 1,2 & 1,2 & 1,8 & 1,7 & 6,3 & 20 & 33,6 & 127,2 & 113,8 \\
\hline Total & 1,4 & 1 & 2,6 & 5,3 & 10,4 & 33 & 46,1 & 93,2 & 137,2 \\
\hline
\end{tabular}

Fonte: SMS/DVIS/Viep/IST-Tabwin/SinanNet. Acesso em 30 jun/20. Sujeito a alteração

Em relação à classificação final dos casos (Tabela 2), observou-se redução do percentual de casos inconclusivos, de 100\% em 2010 para 27\% em 2019. O encerramento dos casos de sífilis adquirida deve ocorrer em 60 dias. Quanto ao diagnóstico final, em $2019,72,5 \%$ dos casos foram confirmados; $27 \%$ inconclusivos; $0,5 \%$ descartado e $0,1 \%$ ignorado/em branco. Em relação a 2018, os casos confirmados aumentaram $4,6 \%$, enquanto que os inconclusivos reduziram 10,9\% (dados não mostrados em tabela). Esses resultados estão relacionados ao fortalecimento das ações de vigilância epidemiológica no município. 
Tabela 2 - Percentual de casos de adquirida por classificação final, Salvador, 2010-2019.

\begin{tabular}{|c|c|c|c|c|c|}
\hline $\begin{array}{c}\text { Ano da } \\
\text { Notificação }\end{array}$ & $\begin{array}{c}\text { Ignorado/ } \\
\text { Branco }\end{array}$ & Confirmado & Descartado & Inconclusivo & Total \\
\hline $\mathbf{2 0 1 0}$ & 0 & 0 & 0 & 100 & 100 \\
\hline $\mathbf{2 0 1 1}$ & 0 & 50 & 1,2 & 48,8 & 100 \\
\hline $\mathbf{2 0 1 2}$ & 0,6 & 27,3 & 0 & 72,1 & 100 \\
\hline $\mathbf{2 0 1 3}$ & 0,3 & 38,3 & 0 & 61,4 & 100 \\
\hline $\mathbf{2 0 1 4}$ & 0,9 & 35,2 & 0,9 & 63,1 & 100 \\
\hline $\mathbf{2 0 1 5}$ & 0,1 & 29,7 & 0,3 & 69,9 & 100 \\
\hline $\mathbf{2 0 1 6}$ & 0,1 & 55 & 0,6 & 44,3 & 100 \\
\hline $\mathbf{2 0 1 7}$ & 0,2 & 73,7 & 0,7 & 25,3 & 100 \\
\hline $\mathbf{2 0 1 8}$ & 0,3 & 69,3 & 0,2 & 30,3 & 100 \\
\hline $\mathbf{2 0 1 9}$ & 0,1 & 72,5 & 0,5 & 27 & 100 \\
\hline
\end{tabular}

Fonte: SMS/DVIS/Viep/IST-Tabwin/SinanNet. Acesso em 30 jun/20. Sujeito a alteração

Analisando a variável raça/cor (Tabela 3), o maior percentual de casos de sífilis adquirida é dos indivíduos que se declaram pardos e pretos. Em 2019, 31,3\% foi de pardos e $23,1 \%$ pretos. Somados, os dois resultados representam $54,4 \%$. O percentual de ignorados/em branco chama a atenção em todos os anos. Verifica-se aumento de 4,4 dessa variável entre 2018 (38,3\%) e 2019 (40\%). Esses dados são fundamentais para a análise da situação de saúde, e formulação de políticas públicas equânimes.

Tabela 3 - Percentual de casos de adquirida por Raça/Cor, Salvador, 2010-2019.

\begin{tabular}{|c|c|c|c|c|c|c|c|}
\hline Ano & $\begin{array}{c}\text { Ignorado/ } \\
\text { Branco }\end{array}$ & Branca & Preta & Amarela & Parda & Indígena & Total \\
\hline $\mathbf{2 0 1 0}$ & 30 & 20 & 30 & 0 & 20 & 0 & 100 \\
\hline $\mathbf{2 0 1 1}$ & 50 & 1,2 & 19,5 & 0 & 29,3 & 0 & 100 \\
\hline $\mathbf{2 0 1 2}$ & 31,2 & 5,2 & 26 & 1,3 & 36,4 & 0 & 100 \\
\hline $\mathbf{2 0 1 3}$ & 22 & 5,2 & 19,7 & 0,3 & 52,8 & 0 & 100 \\
\hline $\mathbf{2 0 1 4}$ & 29,5 & 5,1 & 27 & 0,9 & 37,6 & 0 & 100 \\
\hline $\mathbf{2 0 1 5}$ & 51,2 & 3,3 & 17,9 & 1,1 & 26,3 & 0,2 & 100 \\
\hline $\mathbf{2 0 1 6}$ & 39,2 & 2,8 & 21 & 0,5 & 36,3 & 0,2 & 100 \\
\hline $\mathbf{2 0 1 7}$ & 23,9 & 4 & 28,7 & 1 & 42,1 & 0,3 & 100 \\
\hline $\mathbf{2 0 1 8}$ & 38,3 & 4,1 & 24,9 & 0,6 & 31,8 & 0,3 & 100 \\
\hline $\mathbf{2 0 1 9}$ & 40 & 5 & 23,1 & 0,5 & 31,3 & 0,2 & 100 \\
\hline
\end{tabular}

Fonte: SMS/DVIS/Viep/IST-Tabwin/SinanNet. Acesso em 30 jun/20. Sujeito a alteração 
Em relação à faixa etária (Gráfico 2), os maiores percentuais de detecção da sífilis adquirida estão entre os adultos jovens (20-34 anos) e adultos entre 35 e 49 anos.
Embora esteja alta, observa-se redução da taxa de detecção na faixa etária 20-34 anos de 2018 (47,2/100000 habitantes) para 2019 (45,8/100000 habitantes).

Gráfico 2 - Percentual de casos de sífilis adquirida por Faixa Etária, Salvador, 2010-2019.

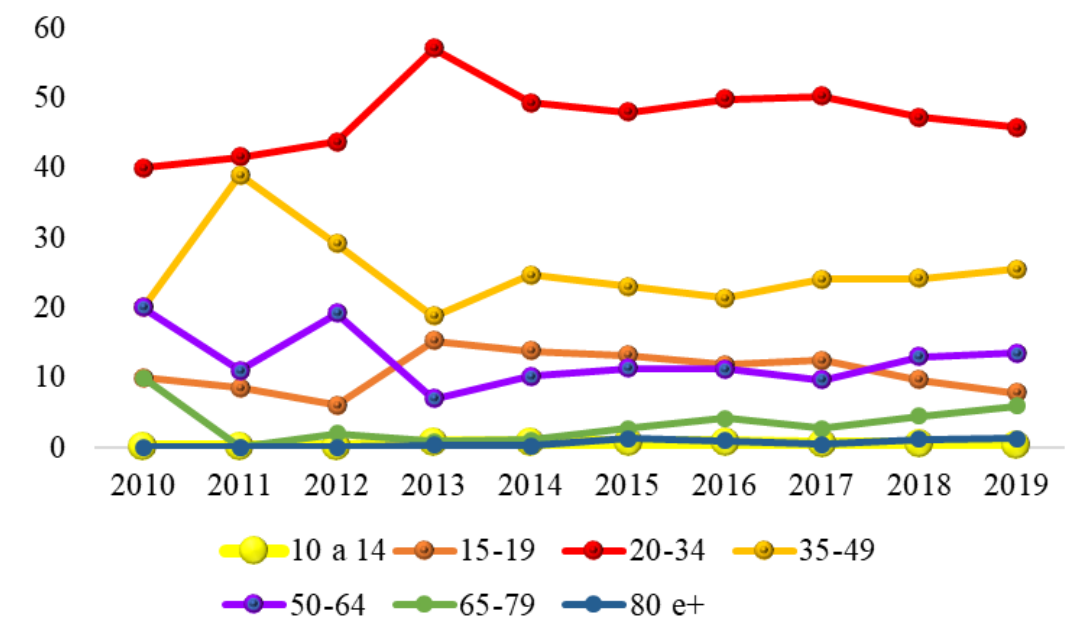

Fonte: SMS/DVIS/Viep/IST-Tabwin/SinanNet. Acesso em 30 jun/20. Sujeito a alteração

De acordo com o Gráfico 3, observa-se que, até 2017, os maiores percentuais de sífilis adquirida se concentravam entre as mulheres. A partir de 2018, os casos entre os homens aumentaram significativamente, passando de 54,4\% em 2017 para 177, 1 em 2018, chegando a 188,1\% em 2019. Esses resultados podem estar relacionados a dois fatores: 1. Mudança nos critérios de caso de sífilis em gestantes, em 2017, quando ficou definido que todas as mulheres diagnosticadas com sífilis durante o pré-natal, parto e/ou puerpério devem ser notificadas como caso de sífilis em gestantes (BRASIL, 2017); 2. Implantação das ações voltadas à Saúde do Homem no município (Sábado do Homem e Novembro Azul).

Gráfico 3 - Taxa de detecção de sífilis adquirida por sexo, Salvador, 2010-2019.

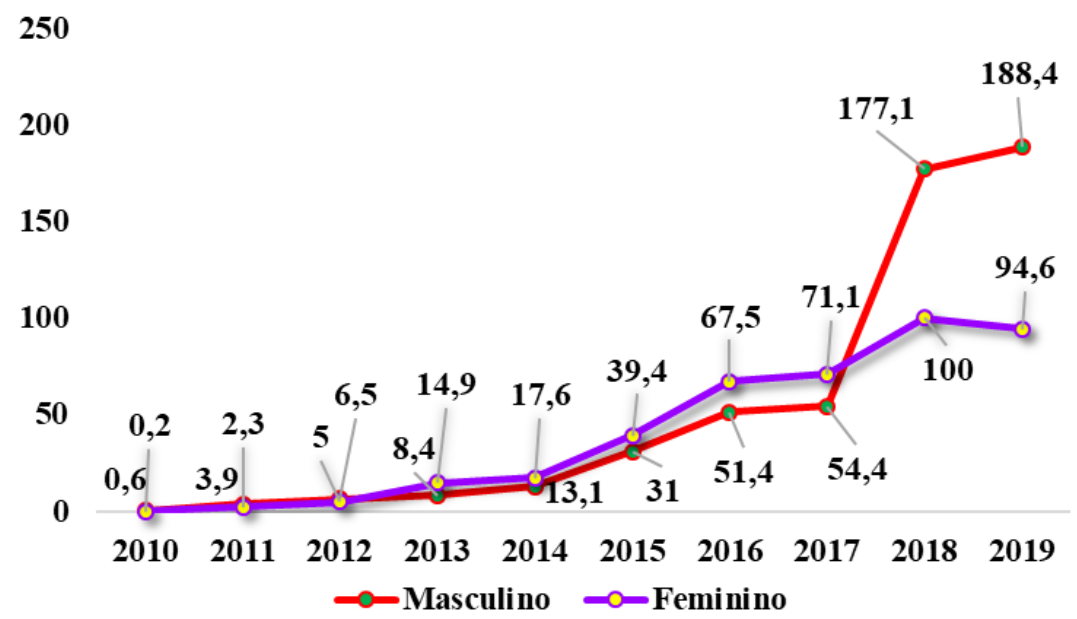

Fonte: SMS/DVIS/Viep/IST-Tabwin/SinanNet. Acesso em 30 jun/20. Sujeito a alteração 
Em todos os anos, na questão relacionada à escolaridade (Gráfico 4) das pessoas com sífilis adquirida, os maiores percentuais são de ignorado/em branco. Fichas de notificação incompletas podem gerar inconsistência das informações e da situação epidemiológica do agravo. De acordo com os dados, o maior percentual de casos de sífilis adquirida é de pessoas com ensino médio completo.

Gráfico 4 - Percentual de casos de sífilis adquirida por nível de escolaridade, Salvador, 2010-2019.

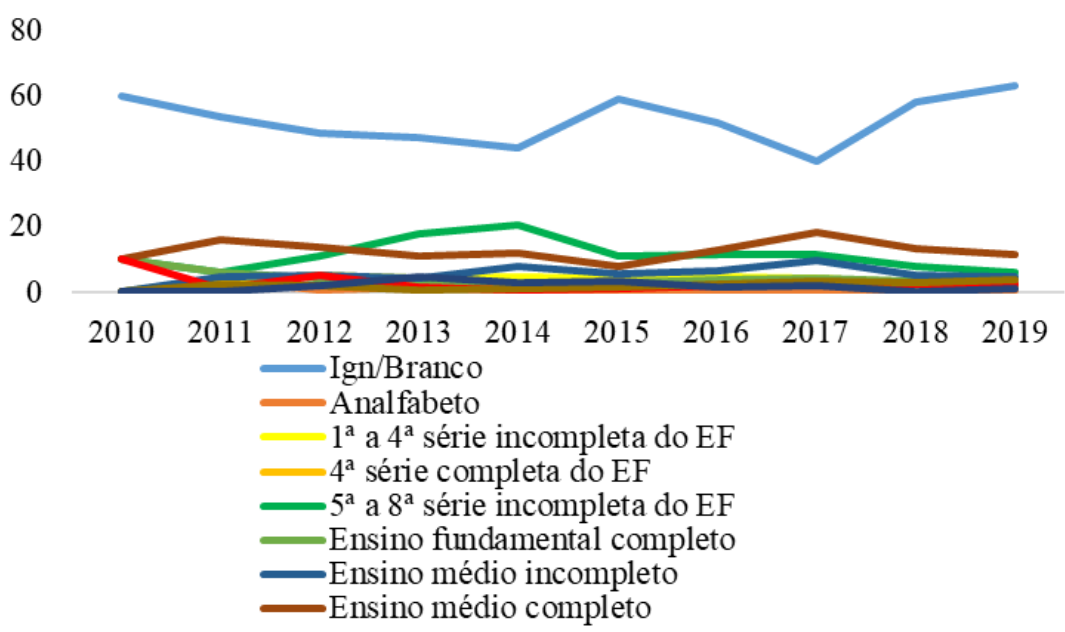

Fonte: SMS/DVIS/Viep/IST-Tabwin/SinanNet. Acesso em 30 jun/20. Sujeito a alteração

Durante o período gestacional, a sífilis pode provocar mortes fetais e neonatais, além de aumentar o risco de morte prematura em crianças (BRASIL, 2018). De 2010 a 2018, foram notificados 7.312 casos de sífilis em gestante em Salvador. Nesse período, a taxa de detecção desse agravo passou de 3,9/1000 nascidos vivos (NV) em 2010 para 36,6/1000 NV em 2019 (Tabela 3). Ressalta-se que os resultados apresentados pelo município estão acima da taxa de detecção do Brasil e da Bahia (BRASIL, 2019; BAHIA, 2019). Comparando 2018 e 2019, observa-se redução da taxa de detecção da sífilis em gestante na maioria dos distritos sanitários (DS), embora estejam altas. Os DS com aumento da taxa de detecção de sífilis em gestante foram São Caetano (74,3/1000NV), Itapagipe (58,2/1000NV) e Subúrbio $(54,1 / 1000 N V)$.

Tabela 3 - Taxa de detecção de sífilis em gestante (1000 NV), por distrito sanitário, Salvador, 2010-2019.

\begin{tabular}{|c|c|c|c|c|c|c|c|c|c|c|}
\hline $\begin{array}{c}\text { Distrito } \\
\text { Sanitário }\end{array}$ & $\mathbf{2 0 1 0}$ & $\mathbf{2 0 1 1}$ & $\mathbf{2 0 1 2}$ & $\mathbf{2 0 1 3}$ & $\mathbf{2 0 1 4}$ & $\mathbf{2 0 1 5}$ & $\mathbf{2 0 1 6}$ & $\mathbf{2 0 1 7}$ & $\mathbf{2 0 1 8}$ & $\mathbf{2 0 1 9}$ \\
\hline $\begin{array}{c}\text { Centro } \\
\text { Histórico }\end{array}$ & 15,7 & 10,4 & 1,2 & 9,9 & 24,1 & 18,2 & 46,9 & 31,9 & 52 & 40,4 \\
\hline $\begin{array}{c}\text { Itapagipe } \\
\text { São }\end{array}$ & 0,5 & 7,9 & 1,6 & 4,5 & 14,3 & 15 & 30,4 & 36,4 & 52,6 & 58,2 \\
\hline $\begin{array}{c}\text { Caetano/ } \\
\text { Valeria }\end{array}$ & 5,3 & 8,8 & 0,8 & 14,2 & 23,5 & 27,3 & 39,6 & 34,7 & 55,3 & 74,3 \\
\hline $\begin{array}{c}\text { Liberdade } \\
\text { Liberda }\end{array}$ & 11,3 & 6,5 & 1 & 22,7 & 22,5 & 17,5 & 29,5 & 29,3 & 43,3 & 34,7 \\
\hline
\end{tabular}




\begin{tabular}{|c|c|c|c|c|c|c|c|c|c|c|}
\hline Brotas & 7 & 7,8 & 1,1 & 16,4 & 15,6 & 6,6 & 11,6 & 14,2 & 27,8 & 18,1 \\
\hline $\begin{array}{c}\text { Barra/R } \\
\text { Vermelho }\end{array}$ & 2,8 & 7,5 & 0,7 & 8,2 & 11,7 & 10,4 & 19,9 & 27,5 & 34,4 & 26,2 \\
\hline $\begin{array}{c}\text { Boca do } \\
\text { Rio }\end{array}$ & 4,6 & 6,4 & 0,4 & 13,7 & 17,7 & 19,6 & 23 & 18,5 & 31,6 & 16,7 \\
\hline Itapuã & 1,7 & 4,2 & 0,3 & 4,9 & 8,5 & 9,6 & 18,2 & 13,8 & 21,1 & 19,7 \\
\hline $\begin{array}{l}\text { Cabula/ } \\
\text { Beiru }\end{array}$ & 2,3 & 7,3 & 1,1 & 10,1 & 11,9 & 13,8 & 20,1 & 20,1 & 42,7 & 35,6 \\
\hline $\begin{array}{l}\text { Pau da } \\
\text { Lima }\end{array}$ & 2,1 & 8 & 1 & 12,6 & 14,8 & 17,2 & 20,5 & 14,7 & 22,7 & 24,2 \\
\hline $\begin{array}{l}\text { Subúrbio } \\
\text { Ferroviário }\end{array}$ & 4,2 & 15,6 & 1,8 & 16,8 & 28,8 & 22,6 & 35,4 & 35,3 & 46,9 & 54,1 \\
\hline Cajazeiras & 4,4 & 8,2 & 0,7 & 4 & 10,5 & 8,7 & 18,8 & 25,1 & 28,8 & 27 \\
\hline Total & 4 & 7,3 & 9,2 & 11,5 & 16,6 & 15,8 & 25,3 & 24,8 & 37,6 & 36,5 \\
\hline
\end{tabular}

Fonte: SMS/DVIS/Viep/IST-Tabwin/SinanNet. Acesso em 30 jun/20. Sujeito a alteração

Em relação à idade (Gráfico 4), os maiores percentuais, entre 2010 e 2019, estão nas faixas etárias entre 20-34 e 15-19 anos.
Em 2019, 68,1\% das gestantes tinham entre 20 e 34 e 19,6\% entre 15 e 19 anos.

Gráfico 4 - Percentual de casos de sífilis em gestante por faixa etária, Salvador, 2010-2019.

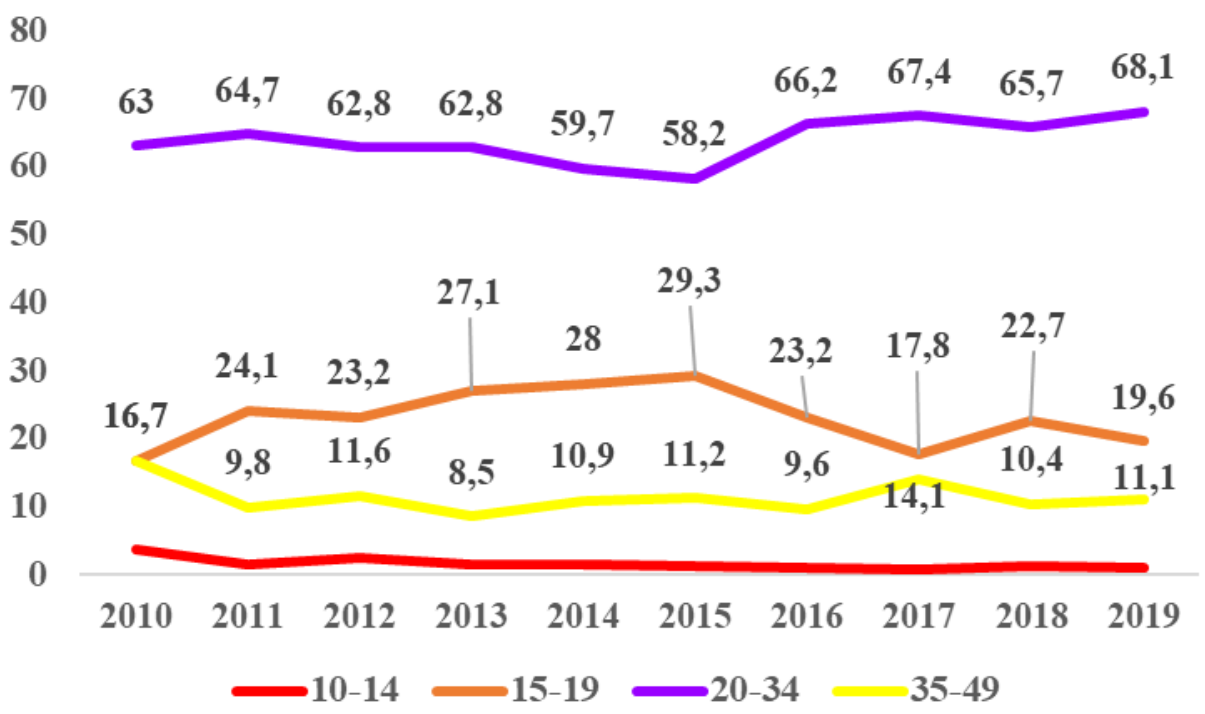

Fonte: SMS/DVIS/Viep/IST-Tabwin/SinanNet. Acesso em 30 jun/20. Sujeito a alteração 
Em todos os anos da série histórica, a cor parda tem os maiores percentuais (Tabela 4). A partir de 2014, houve aumento dos percentuais das pessoas pretas e redução de ignorado/ em branco. Em 2019, 58,6\% das gestantes diagnosticadas com sífilis eram pardas e $26,6 \%$ pretas. Quando consideradas as gestantes pretas e pardas, o percentual passa para $85,2 \%$.

Tabela 4 - Percentual de casos de sífilis em gestante, por raça/cor, Salvador, 2010-2019.

\begin{tabular}{|c|c|c|c|c|c|c|c|}
\hline $\begin{array}{c}\text { Ano da } \\
\text { Notificação }\end{array}$ & $\begin{array}{c}\text { Ignorado/ } \\
\text { Branco }\end{array}$ & Branca & Preta & Amarela & Parda & Indígena & Total \\
\hline $\mathbf{2 0 1 0}$ & 32,7 & 4 & 30,7 & 0,7 & 31,3 & 0,7 & 100 \\
\hline $\mathbf{2 0 1 1}$ & 34,6 & 3,3 & 13 & 0 & 49,1 & 0 & 100 \\
\hline $\mathbf{2 0 1 2}$ & 34,3 & 1,7 & 14 & 0,8 & 49,2 & 0 & 100 \\
\hline $\mathbf{2 0 1 3}$ & 28,6 & 5,2 & 18,6 & 0,2 & 47,4 & 0 & 100 \\
\hline $\mathbf{2 0 1 4}$ & 12,8 & 2,2 & 19,1 & 0,6 & 65,2 & 0 & 100 \\
\hline $\mathbf{2 0 1 5}$ & 8,6 & 4,8 & 27,7 & 0,3 & 58,6 & 0 & 100 \\
\hline $\mathbf{2 0 1 6}$ & 14,7 & 4,8 & 35,5 & 0,7 & 44 & 0,3 & 100 \\
\hline $\mathbf{2 0 1 7}$ & 12,7 & 5 & 36,9 & 2 & 43,4 & 0,1 & 100 \\
\hline $\mathbf{2 0 1 8}$ & 13,7 & 4,5 & 27,8 & 0,9 & 52,7 & 0,4 & 100 \\
\hline $\mathbf{2 0 1 9}$ & 10,4 & 3 & 26,6 & 1,1 & 58,6 & 0,3 & 100 \\
\hline
\end{tabular}

Fonte: SMS/DVIS/Viep/IST-Tabwin/SinanNet. Acesso em 30 jun/20. Sujeito a alteração

Quanto à escolaridade, a informação "ignorado/ branco" apresenta os maiores percentuais em toda a série histórica. Embora tenha sido observada uma diminuição a partir de 2015, os resultados ainda são altos e voltaram a aumentar em 2019.
Entre 2010 e 2019, os maiores percentuais de gestantes com sífilis não concluíram o Ensino Fundamental ou tem ensino médio. Em 2019, 16,8\% das gestantes têm ensino médio e 15,5\% não concluíram o ensino fundamental (Gráfico 5).

Gráfico 5 - Percentual de casos de sífilis em gestante, por nível de escolaridade, Salvador, 2010-2019.

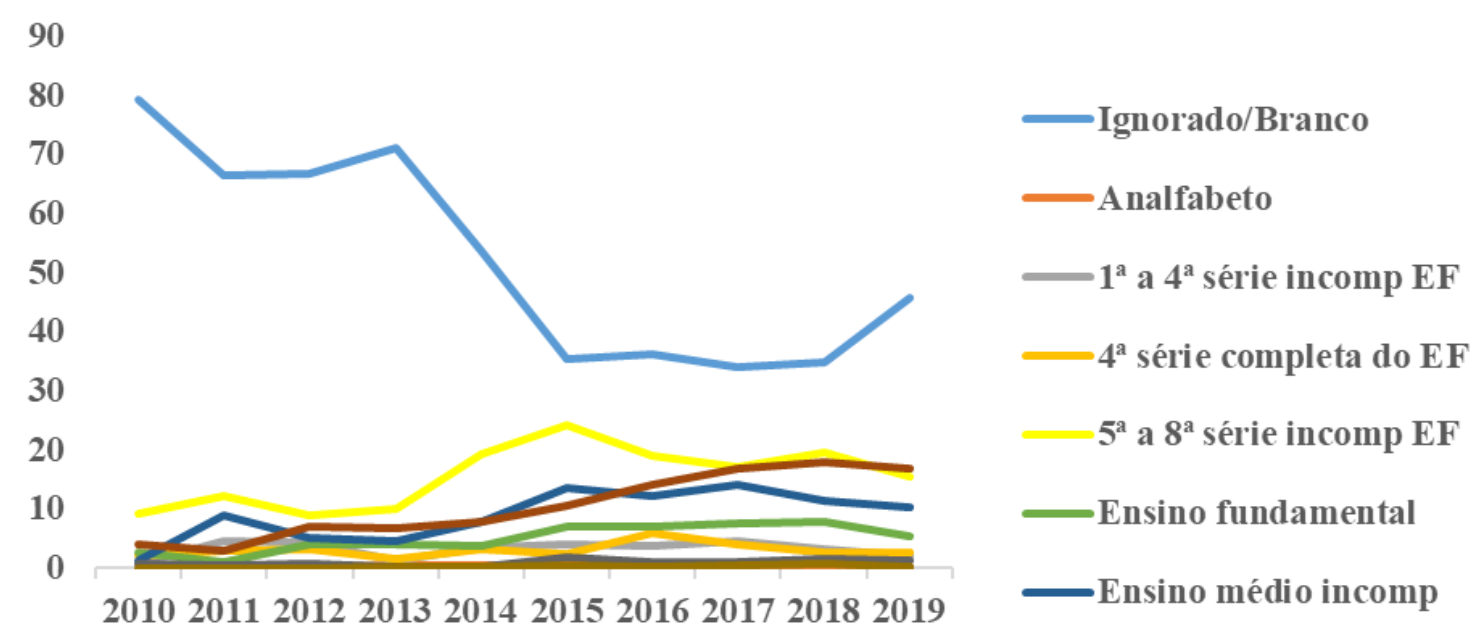

Fonte: SMS/DVIS/Viep/IST-Tabwin/SinanNet. Acesso em 30 jun/20. Sujeito a alteração 
De acordo com o Gráfico 6, até 2018, os maiores percentuais de diagnóstico de sífilis em gestantes se concentraram no terceiro e quarto trimestres. Em 2019 observa-se que os maiores percentuais de diagnóstico de sífilis em gestante são no primeiro (28,8\%) e segundo
$(28,2 \%)$ trimestres. Esse dado pode ser indicativo do fortalecimento das ações de ampliação da testagem rápida para diagnóstico precoce. Destaca-se que o monitoramento, para diagnóstico e tratamento das gestantes, deve ocorrer durante todo o pré-natal.

Gráfico 6 - Percentual de casos de sífilis em gestante, por trimestre do diagnóstico, Salvador, 2010-2019.

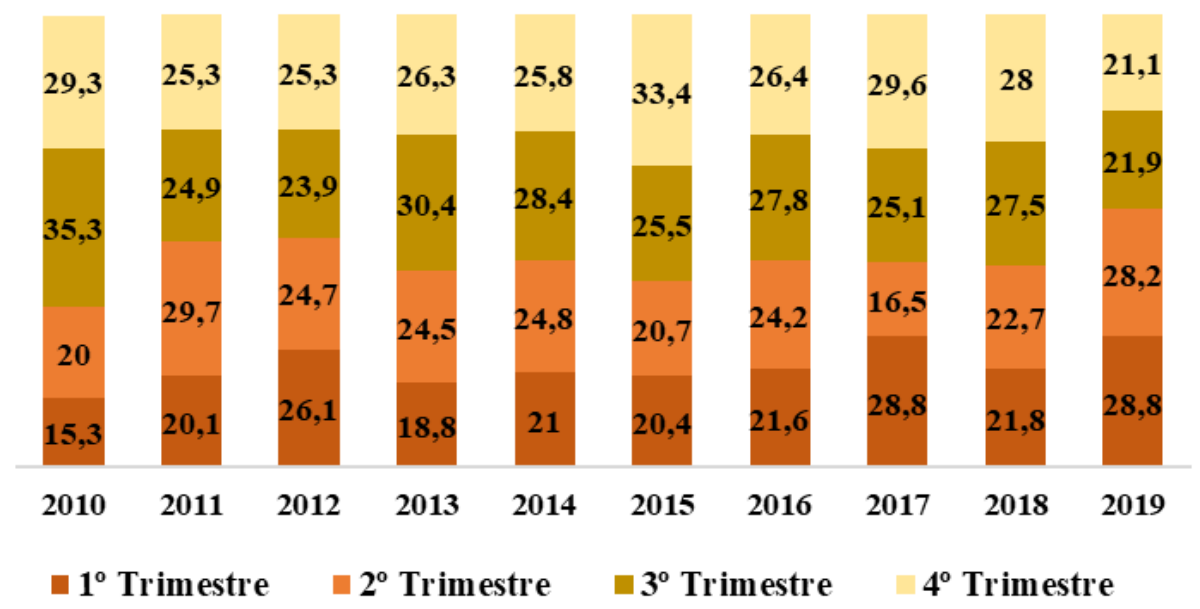

Fonte: SMS/DVIS/Viep/IST-Tabwin/SinanNet. Acesso em 30 jun/20. Sujeito a alteração

Em relação ao esquema de tratamento das gestantes com sífilis, a partir de 2013, o maior percentual se concentra nas três doses de penicilina G Benzatina. Em 2019, foi adotado esse esquema em $67,3 \%$ das gestantes. No entanto, 7,5\% não realizaram o tratamento e 9\% de tratamento está "ignorado/em branco" (Gráfico 7). Esses dados são significativos considerando o risco de transmissão vertical e os impactos da sífilis congênita para mãe-bebê.

Gráfico 7 - Percentual de casos de sífilis em gestante, por esquema de tratamento, Salvador, 2010-2019.

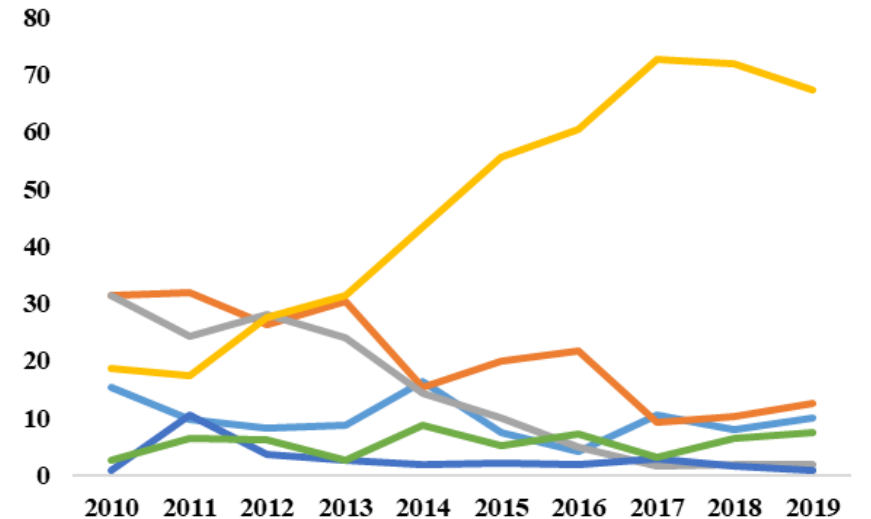

Ignorado/Branco

Penicilina $G$ benzantina 2.400.000 UI

Penicilina G benzantina 4.800.000 UI

Penicilina G benzantina 7.200.000 UI

Outro esquema

- Não realizado

Fonte: SMS/DVIS/Viep/IST-Tabwin/SinanNet. Acesso em 30 jun/20. Sujeito a alteração 
A eliminação da sífilis congênita é multidimensional. Implica a existência de políticas públicas para garantir a qualidade da atenção à gestante e suas parcerias sexuais durante 0 pré-natal e as relações de gênero. Em gestantes não tratadas, a transmissão vertical pode acontecer entre 70\% e 100\% dos casos (BRASIL, 2016). Em 2019, foram notificados 547 casos de sífilis congênita em Salvador - incidência de 16,7/1000 NV. Comparando as taxas de incidência de 2018 (23,7/1000 NV) e 2019 (16,7/1000 NV), verifica-se redução de $29,4 \%$. Ainda é uma taxa alta e indica a importância dos esforços, em todos os níveis do sistema de saúde, para a redução e a eliminação da sífilis congênita. Em contrapartida, esse resultado pode estar relacionado às ações de vigilância epidemiológica, como a identificação dos casos de criança exposta e criança com sífilis congênita. De acordo com o Ministério da Saúde (BRASIL, 2017), os casos de criança exposta devem ter seguimento até os 18 meses, mas não podem ser notificados. Os DS com maiores taxas de incidência (Gráfico 8) foram São Caetano/Naléria, 33,3/1000 NV, (aumento de 14,7\% em relação a 2018); Itapagipe, 31,6/1000 NV, e Subúrbio Ferroviário (28,3/1000 NV).

Gráfico 8 - Taxa de incidência de Sífilis Congênita (1000 NV), por distrito sanitário, Salvador, 2018-2019.

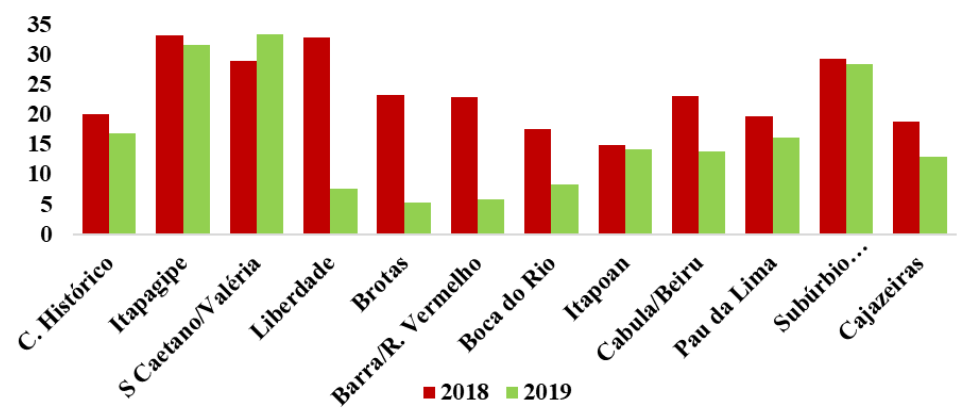

Fonte: SMS/DVIS/Viep/IST-Tabwin/SinanNet. Acesso em 30 jun/20. Sujeito a alteração

Em relação à informação da realização do pré-natal, verificou-se aumento dos casos de sífilis congênita cujas mães realizaram o pré-natal. $O$ acesso ao pré-natal é direito da gestante, e o diagnóstico precoce da sífilis é um dos indicadores da sua qualidade. A triagem pré-natal é obrigatória e fundamental para o diagnóstico precoce e tratamento imediato de gestantes com sífilis e suas parcerias sexuais, independentemente do resultado da testagem (BAHIA, 2019). Esse procedimento visa à prevenção da transmissão vertical da sífilis.

Gráfico 9 - Percentual dos casos de Sífilis Congênita por informação de realização do pré-natal, Salvador, $2010-2019$.

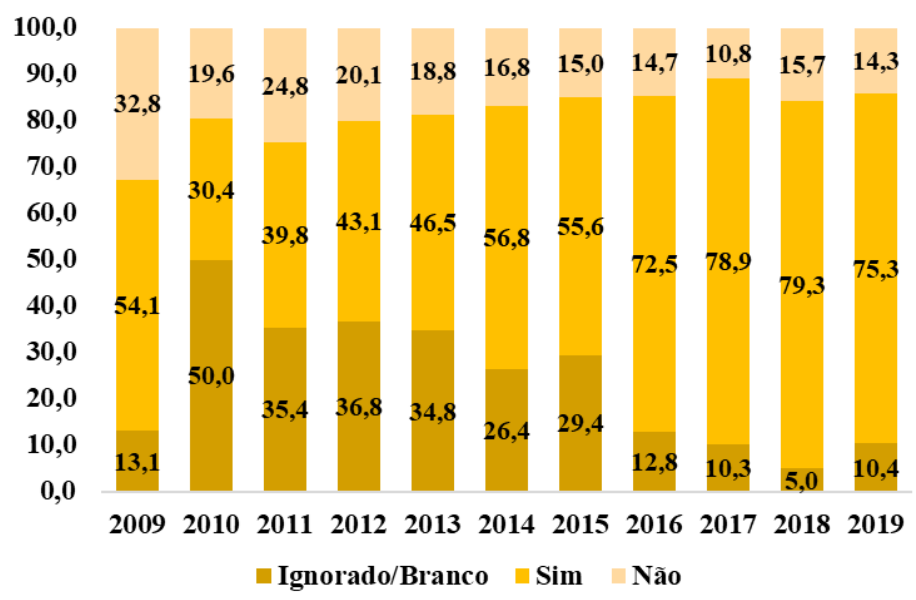

Fonte: SMS/DVIS/Viep/IST-Tabwin/SinanNet. Acesso em 30 jun/20. Sujeito a alteração 
A Tabela 5 indica o percentual de casos segundo o momento do diagnóstico da sífilis materna. Durante o pré-natal, aumentaram de 2010 a 2018. Em 2019, o percentual de 49,5\% representa uma redução de $20,6 \%$ em relação a 2018 (62,4\%), enquanto que o percentual do diagnóstico da sífilis durante o parto/curetagem $(36,4 \%)$ indica um aumento de $57,1 \%$. Esse dado aponta para a importância do monitoramento das gestantes durante o pré-natal para identificar, precocemente, os casos de sífilis em gestante, falhas no tratamento ou reinfecção.

Tabela 5 - Percentual dos casos de Sífilis Congênita segundo momento do diagnóstico da sífilis materna, Salvador, 2010-2019.

\begin{tabular}{|c|c|c|c|c|c|c|}
\hline Ano & $\begin{array}{c}\text { Ignorado/ } \\
\text { Branco }\end{array}$ & Branca & Preta & Amarela & Parda & Total \\
\hline $\mathbf{2 0 0 9}$ & 8,2 & 34,4 & 34,4 & 21,3 & 1,6 & 100 \\
\hline $\mathbf{2 0 1 0}$ & 34,3 & 24,5 & 32,4 & 8,8 & 0 & 100 \\
\hline $\mathbf{2 0 1 1}$ & 22,1 & 22,6 & 38,9 & 15 & 1,3 & 100 \\
\hline $\mathbf{2 0 1 2}$ & 26,9 & 32,9 & 26,9 & 12,7 & 0,7 & 100 \\
\hline $\mathbf{2 0 1 3}$ & 16,8 & 32,8 & 40,3 & 9,8 & 0,5 & 100 \\
\hline $\mathbf{2 0 1 4}$ & 10,4 & 46 & 36,6 & 6 & 0,9 & 100 \\
\hline $\mathbf{2 0 1 5}$ & 9,2 & 41,5 & 43,2 & 5,5 & 0,6 & 100 \\
\hline $\mathbf{2 0 1 6}$ & 10,8 & 51,9 & 28,8 & 8 & 0,5 & 100 \\
\hline $\mathbf{2 0 1 7}$ & 10,1 & 63,2 & 20,2 & 6,1 & 0,4 & 100 \\
\hline $\mathbf{2 0 1 8}$ & 7,1 & 62,4 & 23,2 & 7,1 & 0,2 & 100 \\
\hline $\mathbf{2 0 1 9}$ & 9,1 & 49,5 & 36,4 & 4,6 & 0,4 & 100 \\
\hline
\end{tabular}

Fonte: SMS/DVIS/Viep/IST-Tabwin/SinanNet. Acesso em 30 jun/20. Sujeito a alteração

Após a análise da situação epidemiológica da sífilis em Salvador, foi discutida e aprovada a proposta de estrutura do Plano Municipal de Enfrentamento da Sífilis. Foram adotados os eixos norteadores propostos nos planejamentos do Departamento de Condições Crônicas e IST (DCCl) e do Projeto "Sífilis Não" - Gestão e Governança; Vigilância em Saúde; Cuidado Integral; Comunicação e Educação em Saúde. Cada eixo tem diretrizes, ações estratégicas e seus respectivos responsáveis, numa perspectiva intra e intersetorial.

No plano, está proposto que as diretrizes e ações estratégicas sejam inseridas no Plano Municipal de Saúde (PMS); no Programação Anual de Saúde e na Programação Operacional Anual (POA), para que se possa garantir a institucionalização do Plano da Sífilis como política municipal. Para a execução das atividades do plano, foram previstos os recursos de Incentivo Financeiro às Ações de Vigilância e Prevenção e Controle das DST/Aids e Hepatites Virais.

Para acompanhamento do plano, foram definidos como indicadores a taxa de detecção de sífilis adquirida; a taxa de detecção de sífilis em gestante e a taxa de incidência de sífilis congênita, que deverão ser acompanhados quadrimestralmente com base nos dados fornecidos pelo SINAN; pelo Tabwin; pelo SIM; pelos relatórios mensais do Livro de Acompanhamento de Diagnóstico e Tratamento da Sífilis ("Livro da Sífilis"). Ficou estabelecido que a análise dos indicadores se dará no âmbito da Câmara Técnica Municipal de Prevenção da Transmissão Vertical do HIV, HTLV, Sífilis e Hepatites B e C (CTPTV). 


\section{RESULTADOS}

A experiência, relatada neste estudo para elaboração da proposta do Plano Municipal de Enfrentamento da Sífilis, ganhou dimensões que extrapolaram o processo de planejamento e refletem o nível de compromisso dos atores envolvidos. Dentre os resultados observados nos eixos Gestão e Governança, Vigilância em Saúde, Cuidado Integral e Educomunicação, vale destacar:

\section{Gestão e Governança}

- A proposta do Plano de Enfrentamento da Sífilis foi apresentada em reuniões da CTPTV e do Comitê de Operações Especiais (COE) e encaminhada para o Gabinete do Secretário;

- A ampliação e a consolidação do GT Sífilis possibilitou a implantação da Câmara Técnica Municipal de Prevenção da Transmissão Vertical do HIV, HTLV, Sífilis e Hepatites B e C, cujos membros são representantes dos 12 DS, DVIS e DAS (Portarias 807 e 927/2019).

\section{Vigilância em Saúde}

- Implantação do Protocolo de Investigação dos Casos de Sífilis Congênita em nível da Atenção Primária à Saúde (novembro/2018) e das maternidades (julho/2020);

- Qualificação das notificações dos casos de sífilis congênita realizada nas maternidades, conforme os critérios de caso, para fins de vigilância epidemiológica, definidos pelo Ministério da Saúde - Nota Informativa No 02/2017 (BRASIL, 2017).

\section{Cuidado Integral}

- Visita às maternidades públicas em conjunto com técnicas da Diretoria de Vigilância Epidemiológica Estadual e Distritos Sanitários, a fim de sistematizar os fluxos de investigação e o seguimento dos casos de sífilis congênita e criança exposta.

\section{Educomunicação}

- Capacitação de 394 profissionais de saúde da rede municipal e 25 profissionais do sistema prisional sobre os Protocolos Clínicos e Diretrizes Terapêuticas (PCDT) da sífilis, hepatites virais, HIV e HTLV (2018);

- Intensificação das ações de incentivo ao diagnóstico e tratamento da sífilis em referência ao Dia Nacional de Combate à Sífilis;

- Sessão Pública sobre Sífilis, em conjunto com a Ouvidoria de Saúde, da Câmara de Vereadores de Salvador (outubro/2019), que resultou na instituição do Dia Municipal de Combate à Sífilis;

- II Encontro Municipal sobre as Infecções Sexualmente Transmissíveis, com a participação de 250 profissionais de saúde, entre técnicos e gestores do município e do Estado, e com representantes do Ministério da Saúde (novembro/2019).

Os resultados apontam para um nível crescente de organização e sistematização das ações para o enfrentamento da sífilis em todos os âmbitos da SMS de Salvador - Nível Central, Distritos Sanitários e unidades de saúde. Além disso, também demonstram o nível de engajamento de atores estratégicos à proposta do apoio do Projeto "Sífilis Não".

Do ponto de vista das apoiadoras, foi possível observar aspectos relevantes de um trabalho coletivo, 
intersetorial e multiprofissional, bem como foi uma oportunidade de avaliar o papel do apoiador e a importância das contribuições do Projeto "Sífilis Não" para a implantação/ implementação de ações para combate ao agravo.

\section{CONSIDERAÇÕES FINAIS}

Um dos principais desafios para implementação do Plano diz respeito à sua implantação e implementação como parte de uma política pública de saúde mais ampla. É fundamental articular ações coletivas intra e intersetoriais que impactem os determinantes e condicionantes sociais de saúde e melhorem a qualidade de vida das pessoas. Nesse sentido, foi sugerido que o plano, que atualmente se encontra no Gabinete do Secretário, seja apresentado no Colegiado de Gestão da SMS, submetido à consulta pública, para possibilitar a participação de outros atores estratégicos e seja apresentado ao Conselho Municipal de Saúde (CMS). Após sua validação, que as ações e estratégias sejam inseridas no PMS e nos demais documentos de gestão.

Processo de construção do Plano Municipal de Enfrentamento da Sífilis possibilitou aprendizados diversos para todas as pessoas envolvidas. Em relação ao enfrentamento da sífilis, verificou-se que a redução da sífilis adquirida e em gestantes, assim como a eliminação da sífilis congênita é multidimensional. Implica desde a adoção de políticas públicas para garantir a qualidade da atenção à gestante e suas parcerias sexuais durante o pré-natal, a cultura, sexualidades, identidades de gênero e orientação sexual até as relações de gênero. Quanto ao planejamento em saúde, foi possível identificar a importância da reflexão sobre as práticas adotadas, individual e coletivamente; criar espaços de fala e escuta para poder vislumbrar e propor novos conhecimentos e transformação dos fazeres envolvidos no cuidar.
A experiência de planejamento ascendente pode garantir mais envolvimento dos sujeitos em todas as etapas do trabalho. O sentimento de pertencimento, a identificação com o produto final e a produção de sentidos é a expressão maior das práxis. Nesse processo, o lugar do apoiador pode ser comparado ao de um artesão, que cria estratégias para juntar peças, aparentemente desconexas e disformes, e dá forma a um mosaico, cujos recortes adquirem uma harmonia e dinâmica orgânica de interdependência, em que todos são um. O trabalho é vivo, pois se dá no aqui e no agora de territórios de vida. 


\section{REFERÊNCIAS}

BRANDÃO, C. C.; SCHERER, M. D. dos A. Capacidade de governo em Secretarias Municipais de Saúde. Saúde debate, Rio de Janeiro, v. 43, n. 120, p. 69-83, 2019.

BRASIL. IBGE. Censo Demográfico 2010. Disponível em: http://www.censo2010.ibge.gov. $\mathrm{br} /$ sinopse/index.php?dados=10\&uf=00. Acesso em: 01 nov. 2020.

BRASIL. Ministério da Saúde. Boletim Epidemiológico Sífilis 2020, Brasília, ano VI, n. especial, out. 2020. Disponível em: http://www.aids.gov.br/pt-br/pub/2020/boletimsifilis-2020. Acesso em: 01 nov. 2020.

BRASIL. Ministério da Saúde. Boletim Epidemiológico Sífilis 2019, Brasília, ano V, n. especial, out. 2019. Disponível em: http://www.aids.gov.br/pt-br/pub/2019/boletimepidemiologico-sifilis-2019. Acesso em: 01 nov. 2020.

BRASIL. Ministério da Saúde. Boletim Epidemiológico Sífilis 2018, Brasília, vol. 49, n. 45. 2018. Disponível em: www.aids.gov.br/pub/2018/boletim-epidemiologico-sifilis-2018. Acesso em: 01 nov. 2020.

BRASIL. Ministério da Saúde. Nota Informativa n 02-SEI/2017: DIAHV/SVS/MS. Altera os critérios de definição de casos para notificação de sífilis adquirida, sífilis em gestantes e sífilis congênita. Brasília: Ministério da Saúde, 2017. Disponível em: http://portalsinan. saude.gov.br/images/documentos/Agravos/SifilisGes/Nota_Informativa_Sifilis.pdf. Acesso em: 01 nov. 2020.

BRASIL. Ministério da Saúde. Secretaria de Vigilância em Saúde. Programa Nacional de DST/AIDS. Diretrizes para controle da sífilis congênita: manual de bolso. 2. ed. - Brasília: Ministério da Saúde, 2006. Disponível em: https://bvsms.saude.gov.br/bvs/ publicacoes/manual_sifilis_bolso.pdf. Acesso: 30 out 2020

CAMPOS, G. W. S. Cogestão e neoartesanato: elementos conceituais para repensar o trabalho em saúde combinando responsabilidade e autonomia. Ciênc. Saúde Coletiva, Rio de Janeiro, v. 15, n. 5, p. 2337-2344, ago. 2010. Disponível em: http://www.scielo.br/scielo. php?script=sci_arttext\&pid=S1413-81232010000500009\&lng=en\&nrm=iso. Acesso em: 01 nov. 2020.

CAMPOS, G.W.S. Um método para análise e cogestão de coletivos: a constituição do sujeito, a produção de valor de uso e a democracia em instituições: o Método da Roda. São Paulo: Hucitec. 2000.

CERQUEIRA, S. et al. Guia de planejamento e gestão da SMS Salvador. Salvador: [s.n.], 2020.

PAIM, J. S. Planejamento em saúde para não especialistas. In: CAMPOS, G.W.S. Tratado de Saúde Coletiva. Rio de Janeiro: Hucitec/Fiocruz, 2006. p. 767-782.

SALVADOR (Cidade). Secretaria Municipal da Saúde. Boletim Epidemiológico Sífilis 2020, n. 01 . out. 2020. 
SALVADOR (Cidade). Secretaria Municipal da Saúde. Portaria n 807/2019. Institui a Câmara Técnica Municipal de Prevenção da Transmissão Vertical do HIV, HTLV, Sífilis e Hepatites B e C. Diário Oficial do Município de Salvador, 11 nov. 2019a. Disponível em: https://www.dom.salvador.ba.gov.br/. Acesso em: 01 nov. 2020.

SALVADOR (Cidade). Secretaria Municipal da Saúde. Portaria n 927/2019. Nomeia membros da Câmara Técnica Municipal de Prevenção da Transmissão Vertical do HIV, HTLV, Sífilis e Hepatites B e C. Diário Oficial do Município de Salvador, 11 dez. 2019b. Disponível em: https://www.dom.salvador.ba.gov.br/. Acesso em: 01 nov. 2020.

SALVADOR (Cidade). Secretaria Municipal da Saúde. Plano Municipal de Saúde 20182021. Salvador, BA: Secretaria Municipal da Saúde, 2017. 